\title{
Lock-in and pulsed thermography for solar cells testing
}

\author{
MuZika L. ${ }^{1}$, ŠVANTner M. ${ }^{1}$, KuČERA M. ${ }^{1}$ \\ ${ }^{1}$ New Technologies - Research Centre, University of West Bohemia, Univerzitni 8, 30614 Plzeň, Czech Republic \\ *Corresponding author: msvantne@ntc.zcu.cz
}

Received XX Month XXXX; revised XX Month, XXXX; accepted XX Month XXXX; posted XX Month XXXX (Doc. ID XXXXX); published XX Month XXXX

\begin{abstract}
Inspection of solar cells is an important part of their production process, because even small defects can cause a significant drop of a whole photo-voltaic module performance. LED illuminated lock-in (LEDILIT) and flash-pulse (FPT) thermographic techniques were compared in this study. Lock-in methods are more commonly used for solar cells inspection. The aim of the study was to find out if the FPT is the appropriate method for an inspection of defects of multi-crystalline solar cells. Experimental setup, inspection results and advantages/disadvantages of both methods are presented. It is demonstrated that the LEDILIT is the suitable technique for inspection of defects connected with a photovoltaic effect. Local shunts, cracks as well as artificial laser made defects were detected. Only some of the most significant shunts and laser made defects were identified by the FPT. However, the FPT inspection is much faster than the LEDILIT. The FPT was also able to indicate an inhomogeneity at a bottom layer of a cell, which was not connected with a photo-voltaic effect and which was not revealed by the LEDILIT.
\end{abstract}

OCIS codes: (120.4290) Nondestructive testing; (110.6820) Thermal imaging; (110.3080) Infrared imaging; (350.6050) Solar energy.

http://dx.doi.org/

\section{INTRODUCTION}

Energy from renewable sources is becoming increasingly important in energy industry. One of the most important renewable sources is solar energy converted by solar cells into electricity. Energy obtained from solar cells is still growing [1] and it is therefore of high importance to produce high quality cells. A lot of methods were developed for solar industry component testing and inspection, which are used in research, production process or for a diagnostics at their operation [2]. These inspection processes help to ensure and track required quality of individual components in photovoltaic industry at different stages of their life cycle.

This study is focused on solar cells testing. Defects can arise at different stages of solar cells life cycle. They can be induced, for example, by non-suitable manufacturing parameters, inappropriate transport or at their manipulation during a production of solar modules. Solar cells are composed by several layers and defects can occur in each of them.

The basis of cell is a PN junction. Under an illumination of a top side of the cell by photons of suitable wavelength, due to absorption of photons in the semiconductor material, a photocurrent is induced [3]. This process is called a photo-voltaic effect. A damage of the PN junction can cause severe drop of electrical performance. The current induced in the PN junction is collected by contacts (electrodes). There are top and bottom contacts in solar cells. The top contacts are composed by main conductors (busbars) and smaller conductors (fingers) perpendicular to the busbars. The contacts create a metallic grid. The denser the grid is the better drains electrons from the cell but at the same time it reduces the active area of the cell. The bottom contacts are made from a one piece of a conductor. A damage of the electrodes can deteriorate current collection and a performance of a cell can then decrease. An antireflection coating is on the top of the cell. A common composition of a solar cell is shown in Fig. 1.

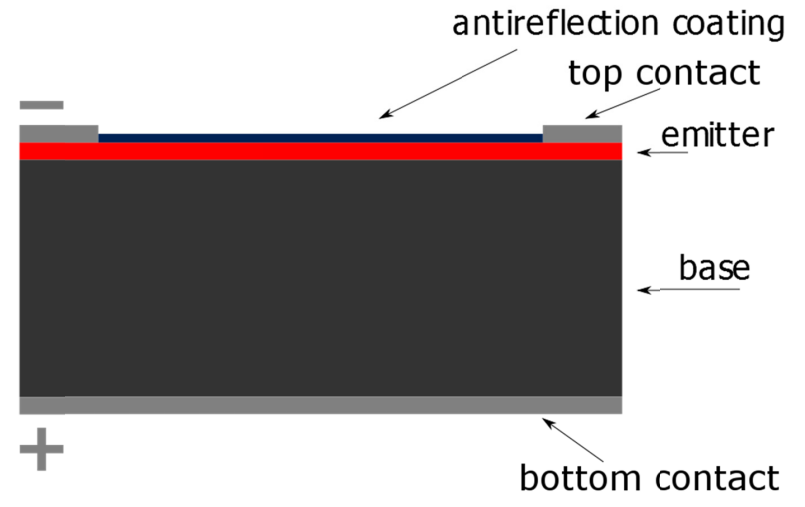

Fig. 1. A general structure of a solar cell.

Solar cells are mostly divided into mono-crystalline, multicrystalline (polycrystalline) or thin film based cells. Some types of defects are typical for a particular type of solar cells. For example, delamination, cracks, passivation degradation, light-induced degradation, solder corrosion, finger failure or humidity corrosion are described in [4], shunts and their detection are solved in [5]. 
There is not a universal method enabling testing of all types of defects. Very popular are techniques based on current-voltage characteristics measurement. This type of testing allows determination of electric parameters of a measured cell [6]. These methods can easily determine a damage of a solar cell. Fast measurement time of these methods makes them popular for a use in a serial production process. However, the current-voltage methods cannot spatially locate defects and, therefore, are not suitable for a more detailed analysis. Their other disadvantage is that they are contact methods. Contacting is a complication in an inspection procedure and represents a possible risk, because solar cells are usually very thin and brittle and can be damaged during the testing.

Different inspection methods are used for a spatial location of defects, however, most of them also need contacting of tested cells. Electroluminescence methods, which advantages and disadvantages are described for example in [7], belong to the most popular. These methods are fast enough and have good spatial resolution. However, they also need contacting and can be used on cells with a grid structure (an electrical conductor) only [7].

Active thermography based methods are a different group of inspection methods often used for solar cells testing [3][5][8]. Active thermography methods [9] use an external excitation source to induce a thermal process in a tested sample. A thermal response of the sample is then measured by a thermographic camera. The response is reflected by small temperature changes of a tested sample surface. These changes are related to local differences of thermal properties, surface and subsurface defects or concentration of local heat sources. Different excitation sources and procedures can be used for different applications. The commonly used excitation sources are for example flash lamps, halogen lamps, ultrasound, hot air or electric current. The common methods are pulsed, lock-in and step excitation. These methods are called thermographic testing or infrared testing [10]. The thermographic testing is used in a lot of applications, often for composite structures inspection [11], but for example also for optical components [12].

Lock-in thermographic methods are commonly used for solar cells inspection as well. These methods are based on a periodical excitation of a measured sample with a lock-in frequency and temperature response recording by a thermographic camera. The response is than processed with one of the lock-in algorithm, which allows noise reduction and improves detectability of defects. More about lock-in processing can be found in [13] or more in detail in book [14]. There are several lock-in techniques used for solar cells testing. Most of them use a light source for the excitation - a light illumination of a measured sample. The light source should have a specification close to sun irradiation parameters (a wavelength range and intensity). These methods are called as ILIT (illuminated lock-in thermography) and their approaches differ based on a tested solar cell state (for example open circuit or short circuit). Other thermographic lock-in inspection methods use an electric current for the excitation. A comparison of different lock-in thermography methods for solar cells testing is described for example in [5].

Different lock-in thermography approaches can be used based on inspection requirements and type of defects, which should be found. Lock-in thermography can be in solar cells applications [15] used for example for an analysis of weak ohmic shunts, strong ohmic shunts, bulk defects in cells, cracks, local I-V characteristic or breakdowns. However, even if thermography is in principle a noncontact method, many of lock-in thermographic methods for solar cells testing need a contacting of the cells. That means these techniques have similar constrictions and disadvantages as the electroluminescence methods. Noncontact lock-in approaches can be used even for cells without an electrode structure and the cells are in this case open circuited. These techniques are therefore often called Voc-ILIT (open circuit voltage illuminated lock-in thermography) [16]. The main disadvantage of the lock-in thermography is a longer inspection time - an inspection takes mostly from several minutes to tens of minutes [3][17] and these methods are therefore not suitable for serial testing.

Pulsed thermography [18][19] is another very popular active thermography method. A tested sample is excited with one very short pulse, which length is typically a few milliseconds. A thermal response of the sample is measured by an infrared camera, which should be a high speed and high sensitivity camera in the most cases. The pulsed thermography using a flash lamp as the excitation source (flash-pulse thermography) is a suitable method for an inspection of different materials. The inspection depths are from a top surface up to several millimeters under the surface [20]. The temperature response is mostly processed by different algorithms to suppress noise and improve a detectability of defects. More about algorithms for postprocessing used in pulsed thermography can be found for example in [21] or [22].

The pulsed thermographic testing is well established in a lot of applications (composites testing for example [18]) due to its short measurement time and noncontact nature. However, it is commonly not used for solar cells testing. This experimental work was focused on the analysis of possibilities of flash-pulse thermographic methods for a solar cells inspection. The use of flash-pulse thermographic methods would keep the advantage of noncontact measurement (no need of a contacting) and it could significantly shorten a measurement time compared to the lock-in thermography. Thus, in this work, the inspection experiments using both methods were performed, the results were analyzed, a usability of the flash pulse thermographic methods was discussed and some conclusions were made including an explanation of basic differences between the methods.

A comparison of the FPT and LEDLIT methods was made using artificially made defects. The defined defects were made by laser technology, which allowed creation of different types of defects at defined positions. The defects were made by a different laser beam intensity and so it was possible to make defects of a different significance (a different damage level). This approach enabled an easy comparison of both thermographic methods.

\section{EXPERIMENTAL SETUP}

Results of flash-pulse and LED lock-in thermographic inspections of solar cells were compared in this work. Artificial defects were made on the tested solar cells by a laser. The thermographic inspections were performed before and after the defects were made. Relations between thermographic inspection results and some of found defects were shown on current-voltage characteristics (IVC) measurement.

Thermographic methods are in general a tool for finding interesting spots, often called as indications, which physical origins should be further investigated [5]. These investigations were not a subject of this research. However, to avoid confusions, we have in this paper assigned probable defect types to indications, which did not come from the known artificial laser made defects.

\section{A. Experimental samples}

The experiments were performed on multi-crystalline solar cells. Three samples labeled as solar cell no. 1-3 were used. Artificial defects were created on the top side of the cells no. 1 and 2 using a pulsed laser SPI G3-HS $20 \mathrm{~W}$ [23] with scanning head Scancube 10 with f-theta objective ( $\mathrm{f}=160 \mathrm{~mm}$ ). The defects are illustrated in Fig. 2.

The defect on the cell no. 1 was in the form of a line parallel to busbars going across the entire cell in its center. The defect was made using the laser parameters: $100 \%$ power, speed $100 \mathrm{~mm} / \mathrm{s}$, pulse- 
length $200 \mathrm{~ns}$ and scanning frequency $25 \mathrm{kHz}$. The defect interrupted all fingers. This damage caused disruption of the grid, which collects electrons from the cell. Thus, a worse solar cell performance could be expected. A defect, which interrupts all fingers, doesn't belong to typical defects of solar cells. But finger damages are common and can be caused for example by a soldering (manufacturing process) or cracks [4]. The defect, which goes across the entire cell, should be easily detectable for both used active thermography techniques due to the size of the defect and due to its expected effect on a performance decay of the cell.

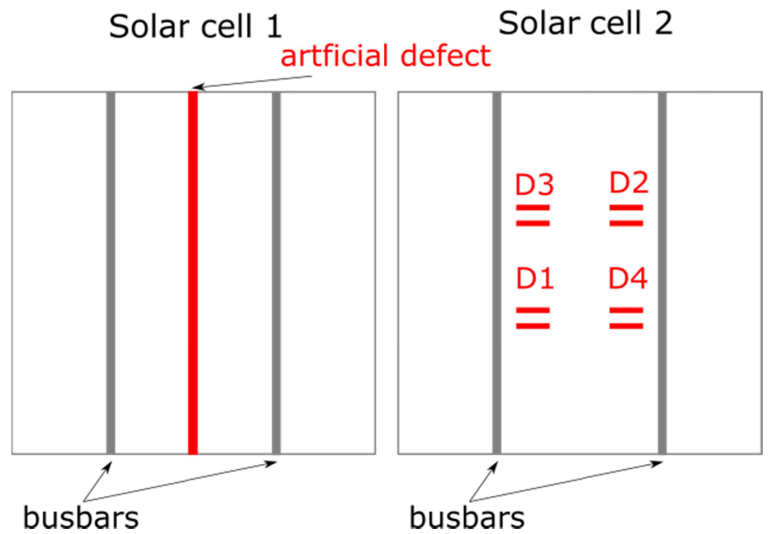

Fig. 2 Schematic illustration of artificial defects and their locations on the solar cells no. 1 and no. 2 .

Multiple defects by different laser parameters were created on the cell no. 2. The defects, which location is shown in Fig. 2, were labeled D1-D4 and the used laser parameters are listed in Tab.1. The laser parameters sets determine so called laser heat input [23], which is one of the most important parameters defining an effect of laser processing on a surface. The highest heat input was used for D1 and D2 defects. The lowest heat input was used for D3 and D4 defects. These defects damaged the emitter of the cell and could simulate for example cracks. The defects D1-D4 are significantly smaller than the defect created on the cell no.1, therefore, a worse detectability of these defects was expected.

Tab. 1 Laser parameters table

\begin{tabular}{cccccc}
\hline defect & $\begin{array}{c}\text { power } \\
(\%)\end{array}$ & $\begin{array}{c}\text { scanning } \\
\text { speed } \\
(\mathrm{mm} / \mathrm{s})\end{array}$ & $\begin{array}{c}\text { frequency } \\
(\mathrm{kHz})\end{array}$ & $\begin{array}{c}\text { pulse } \\
\text { length } \\
(\mathrm{ns})\end{array}$ & $\begin{array}{c}\text { heat } \\
\text { input } \\
(\mathrm{mJ} \cdot \mathrm{s} / \mathrm{m})\end{array}$ \\
\hline D1 & 100 & 100 & 25 & 200 & 8 \\
D2 & 100 & 200 & 25 & 200 & 4 \\
D3 & 100 & 800 & 25 & 200 & 1 \\
D4 & 100 & 100 & 66 & 65 & 3 \\
\hline
\end{tabular}

IVC of the cells no. 1 and 2 were measured to determine a severity of the laser-generated defects. Thermographic inspections and IVC measurements were performed both on the original solar cells and after the creation the defects.

The solar cell no. 3 was of the same type as the cells no. 1 and 2, however, there was a different producer. It was not artificially damaged. However, there was an original inhomogeneity in a form of a darker area in the bottom side of the cell. The origin of this inhomogeneity was not fully clear. We assumed that the cell soaked up with some liquid. The cells are transported in transport boxes, where they are placed on each other and they are usually separated by paper after some count of cells. The cell no. 3 was on the top and a separating paper under this cell was soaked up as well. The origin of this inhomogeneity was probably connected with a transportation or storage processes. The goal of thermographic inspections of this cell was to determine, if this inhomogeneity can be indicated, and the IVC was not performed in this case.

\section{B. Flash Pulse inspection}

The flash-pulse thermographic inspections were performed with an infrared non-destructive testing system by Automation Technology (AT). The flash-pulse experimental setup is shown in Fig. 3. The measurement configuration consisted of a flash lamp with a highpower flash-pulse generator of maximum output power $6 \mathrm{~kJ}$ (the excitation source), high-speed high-sensitivity cooled infrared camera FLIR SC7650, synchronization and control unit and a control PC with a control and evaluation software. The software allowed setting of inspection parameters, performing a test and evaluation the flashpulse measurement by different methods.

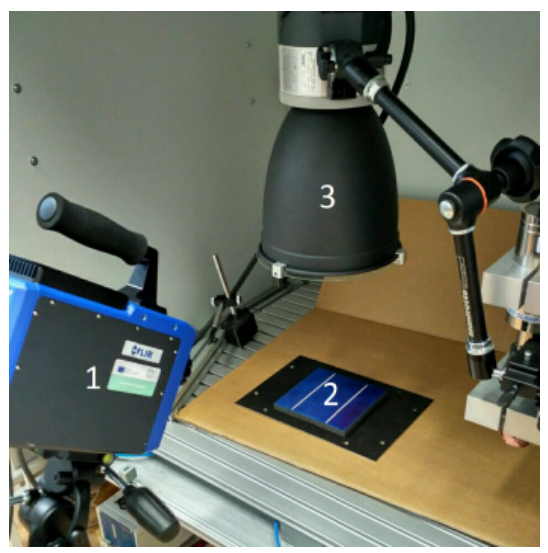

Fig. 3 Flash-pulse thermographic inspection configuration: the infrared camera (1), a tested sample (2) and the flash lamp - the flash light excitation source (3).

A full power flash pulse (the pulse length was about 3-5 ms) was used for the excitation. The sampling rate of the infrared camera was $100 \mathrm{~Hz}$ and the data acquisition time (i.e. the response signal recording time after the excitation pulse) was $10 \mathrm{~s}$. The excitation (flash illumination) of all samples and the detection of their thermal response were performed at the top-side of the tested cells layer with the metallic grid), that means a reflection FPT configuration was used.

Three evaluation methods were offered by the AT software: RootModel, e-Model and Pulse-Phase evaluation. The Root-Model and the eModel are based on an approximation of a measured signal of each pixel by a synthetic function (different type of polynomials). This procedure is used for derivatives calculation, which can be visualized in different forms. The Pulse-Phase evaluation is based on the Fast Fourier Transform of the measured signal of each pixel. Amplitude or phase spectrum data as functions of frequency can be evaluated and visualized. The Pulse-Phase method is described more in detail for example in [24]. The software allows a flexible switching between the individual methods. Thus, different evaluation methods, evaluation parameters (data range, offset image subtraction, polynomial order, etc.) and results representation approaches (amplitude, phase, $1^{\text {st }}$ derivative, etc.) are tested at each measurement and the most appropriate evaluation procedure is used to obtain the best results.

\section{LED lock-in inspection}


LED lock-in thermographic inspections were also performed with the AT infrared non-destructive testing system, however, a self-made LED reflector was used for the excitation of the tested samples. The LED lock-in experimental setup is shown in Fig. 4. The measurement configuration consisted of LED reflector composed of 196 LED diodes of different wavelengths with an overall performance $1.2 \mathrm{~W}$, highspeed high-sensitivity cooled infrared camera FLIR SC7650, synchronization and control unit and a control PC with a control and evaluation software. The AT system allows to connect external excitation sources. The LED reflector could be therefore connected to and controlled by the AT testing system, which allowed setting of inspection parameters, performing a test and evaluation of the lock-in measurement by different methods.

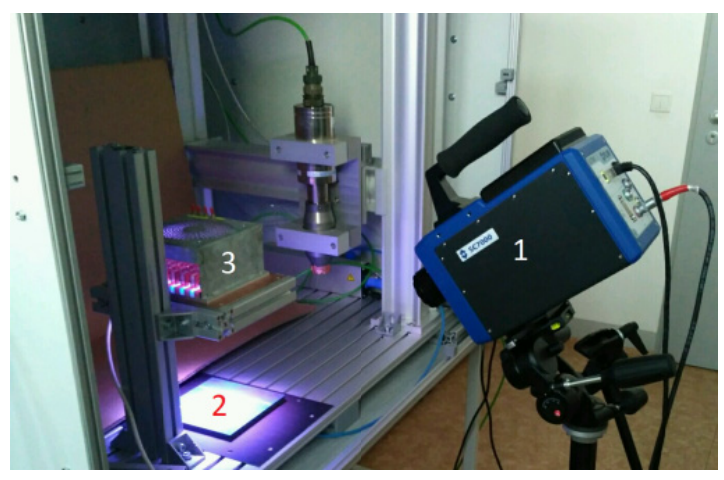

Fig. 4 LED lock-in thermographic inspection configuration: the infrared camera (1), a tested sample (2), LED reflector - excitation source (3).

The excitation of the sample was performed periodically using a sinus shaped profile of the excitation power. One period took $3 \mathrm{~s}$ and one test took about 7 minutes. Sampling rate of the infrared camera was $50 \mathrm{~Hz}$ and a tested sample response was recorded and evaluated throughout the whole excitation procedure. The excitation (LED light illumination) of all samples and the detection of their thermal response were performed at the top-side of the tested cells (layer with the metallic grid), that means a reflection LEDILIT configuration was used.

The control and evaluation software by AT offers several lock-in evaluation techniques. A lock-in online module developed by AT was used for the response evaluation. Several methods are available for lock-in online evaluation by the AT software: "Average" (based on calculation of mean values), "Single Phase Reference" (based on correlation between a reference signal and thermal response) or "Dual Phase Reference" (DPR). The DPR was used for the evaluation of these experiments. This method is also based on a correlation between a reference signal and thermal response. However, two reference signals are used in this case: the original reference signal and a signal created from the original one by its $\pi / 2$ shift. The use of the two reference signals then allows to calculate both phase and amplitude images. This method is similar to the online two channel correlation method described in [14]. For the evaluations made in this work, an excitation control signal was used as the reference signal.

\section{Current-voltage characteristic measurement}

The IVC tests were made to verify a functionality of the tested solar cells and to quantify a connection between the created defects and their influence on the tested cells performance. A Forward Bias I-V Illuminated method [25] was used. The same LED light source as for the LEDILIT tests was used for the illumination, which intensity was about $80 \mathrm{~W} / \mathrm{m}^{2}$. Each IVC was measured three times and averaged to obtain a resulting I-V curve. The maximum power of the cells was evaluated by the IVC measurement results.
The IVCs were determined for the solar cells no. 1 and 2. The IVC measurements were not performed according to IEC 60904-3 standard. Thus, the results did not represent a real standardized performance of the tested cells, but the results were only for a comparative illustration of an influence of the defects created by the laser in this work.

\section{RESULTS}

\section{A. Solar cell 1}

Results of the FPT and LEDILIT inspections of the cell no. 1 are presented in Fig. 5. The FPT defectograms were obtained by the pulsephase evaluation method and the phase results are presented. The LEDILIT defectograms were obtained by the DPR method and phase results presentation.

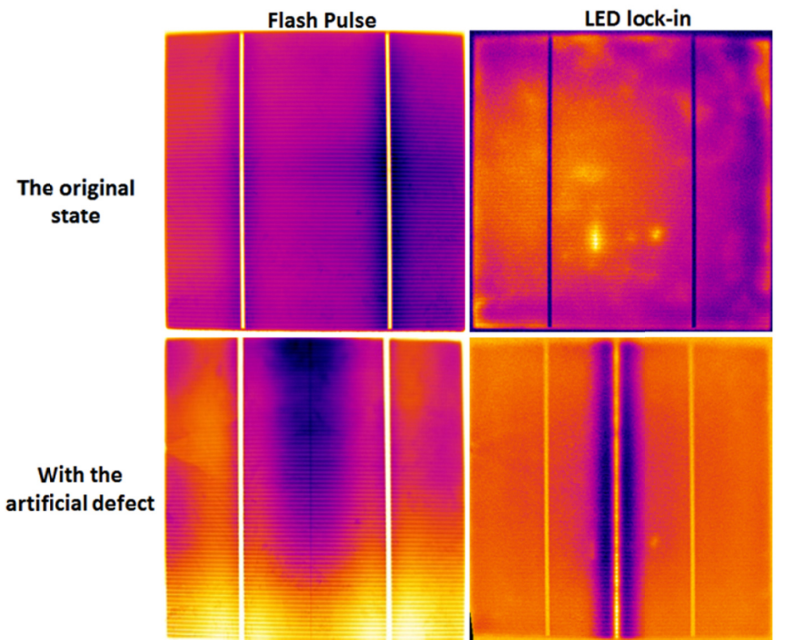

Fig. 5 Results of FPT (phase results) and LEDILIT (DPR, phase results) inspections of the solar cell no. 1 before and after the creation of the artificial defect by the laser.

The LEDILIT inspection of the original cell (no. 1) showed several distinct indications between the busbars in the central part of the cell. Similar indications were called as shunts in [5]. These shunts were present on the original cell and they were apparently formed during production and/or subsequent manipulation processes. The shunts were not found by the FPT inspection. A difference between the right side of the cell and the rest of the cell was evident by the LEDILIT results. A part of the cell to the right of the right busbar is darker than the rest of the cell. A similar effect was also observed by the FPT. However, the FPT showed a distinct contrast (i.e. color difference) of a surroundings of the right busbar. It was however not possible to explain these effects without additional analyses.

The defect made by the laser on the cell no. 1 reduced its performance considerably, how it was evident from the IVC curves showed in Fig. 6. The maximum power of the original cell (at the given illumination) was $358 \mathrm{~mW}$, the laser made defect caused a decrease of its power to $268 \mathrm{~mW}$ (i.e. the power drop by about $25 \%$ ). The defect had a very clear indication by the LEDILIT inspection and was clearly detectable along its entire length. It was so contrasting that it overlapped most of the local shunts found on the original cell. The defect was also found by the FPT inspection. However, it was not as clear as in the case of the LEDILIT inspection.

A bright area at the bottom part of the FPT defectogram was also observed. This was probably caused by an illumination (excitation) 
inhomogeneity due to a geometrical set-up and/or flash-lamp properties.

Differences between the LEDILIT and FPT results were caused by different thermal processes, which were induced by the LEDILIT and FPT excitation, respectively. The indications of the defects at the flashpulse excitation were mostly a result of a direct thermal response (the defects acted as a discontinuity and/or inhomogeneity in the induced thermal process). Thus, the artificial defect made over the entire height of the cell was detected, but the small local defects were not indicated. The induced photo voltaic effect played a more significant role in the case of the LEDILIT inspection. A thermal response of the defects, which influenced the photo-voltaic effect, was enhanced by residual currents. As a result, the small local shunts were indicated by the LEDILIT inspection on the original cell. Also the indication of the laser made artificial defect was much more clearly indicated by the LEDILIT, because the defect (fingers damage) influenced currents in the solar cell. The small shunts were not visible on the cell with the laser made defect, because their indications were much weaker in comparison with the indication caused by the laser made defect.

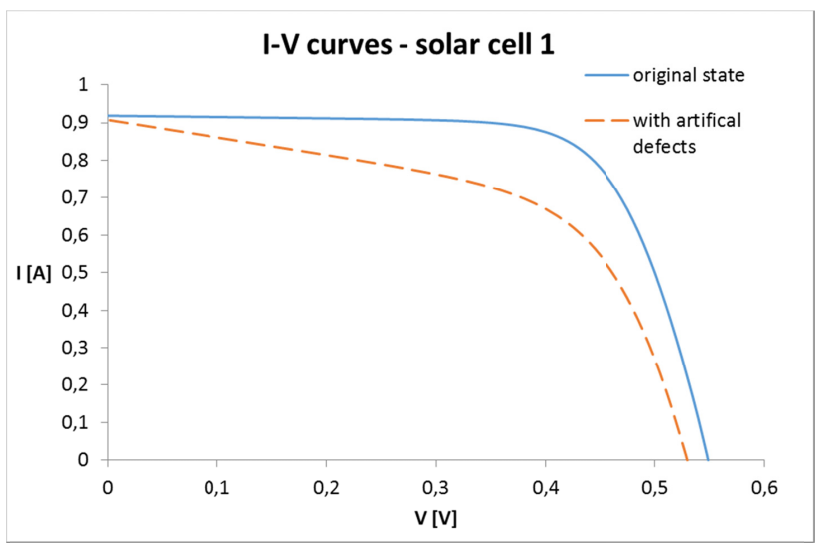

Fig. 6 Current-voltage curves of the tested solar cell no. 1 before and after the artificial defect was made.

\section{B. Solar cell 2}

Results of the FPT and LEDILIT inspections of the cell no. 2 are presented in Fig. 7. The evaluation procedures were the same as for the cell no. 1.

The LEDILIT inspection of the original cell no. 2 showed 2 line indications (white arrows in Fig. 7). These indications are most certainly cracks [4], probably caused by a mechanical load. A number of local shunts can be also identified on the defectogram. The inspection showed that the cell no. 2 looked more damaged (in original state) than the cell no. 1 . This corresponded to the IVC measurement results, which are shown in Fig. 8 for the cell no. 2. The maximum power of the original cell no. $2(347 \mathrm{~mW})$ was lower than the maximum power of the cell no. 1 (358 $\mathrm{mW})$. This difference was however only about $3 \%$ and it seemed to be not so significant.

The FPT inspection did not detected the cracks and most of the local shunts found out by the LEDILIT. However, some of the most significant shunt indications were also visible on the FPT defectogram. It should be mentioned, that the shunt indications can in some cases represent grains, which are typical for multi-crystalline solar cells. These grains have sometimes a different emissivity and so can be identified by thermographic methods (both LEDILIT and FPT). Very bright (overburned/out of range) regions also showed the above mentioned problem with an inhomogeneity of the flash illumination.
The defects made by the laser on the cell no. 2 were clearly indicated by the LEDILIT inspection. The original cracks and local shunt were mostly still visible, but much less distinct because of a high intensity of the indications of the laser made defect. That indicated a greater influence of the laser made defects, which was confirmed also by the IVC measurement. The maximum power of the cell no. 2 after the creation of the laser defects was $315 \mathrm{~mW}$. The laser made defects reduced the maximum power of about $9 \%$ compared to the original cell. That implied their influence was more significant than the cracks and local shunts found on the original cell, but not as strong as the defect made on the cell no. 1 . This conclusion was also confirmed by the comparison of IVC measurement (maximum power) of both cells before and after the creation of the laser made defects.

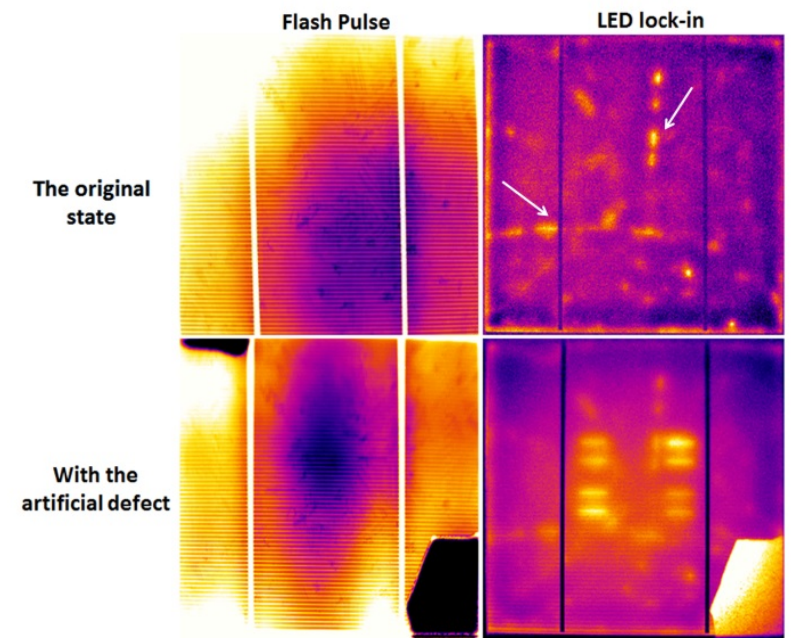

Fig. 7 Results of FPT (phase results) and LEDILIT (DPR phase results) inspections of the solar cell no. 2 before and after the creation of the artificial defect by the laser. White arrows by LEDLIT (original image) point to cracks.

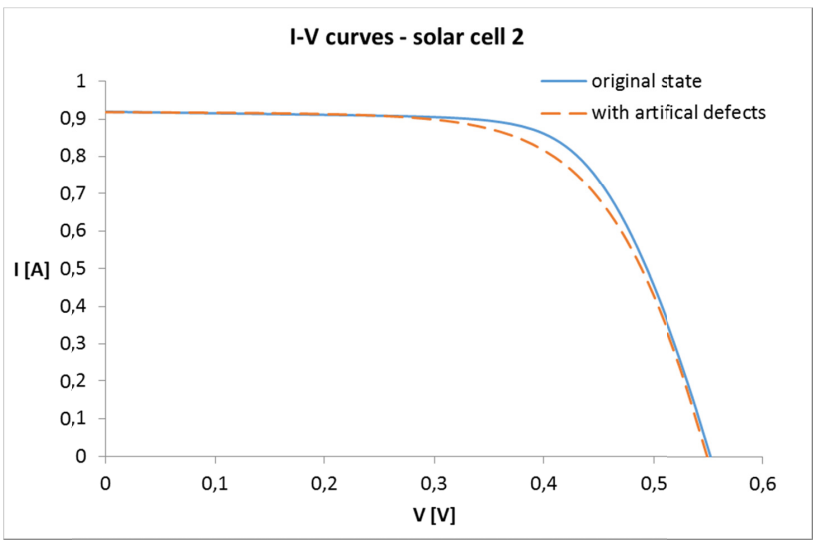

Fig. 8 Current-voltage curves of the tested solar cell no. 2 before and after the artificial defect was made.

It could be also seen on the LEDILIT defectogram of the cell no. 2 with the laser made defects (Fig. 7, the right down defectogram), that the defects D1 (CNR 7.9 dB) and D2 (CNR 7.5 dB) were more distinct compared to the defect D3 (CNR $7.2 \mathrm{~dB}$ ) and D4 (CNR $5.7 \mathrm{~dB})$. This observation corresponded with the assumptions that higher laser heat input used for the defects D1 and D2 caused a higher damage.

Defects D1 and D2 could be also found on the FPT defectogram. It showed that these defects were created with the higher heat input and 
therefore were more significant and detectable also by the FPT. The indications of these defects were very weak and partly disappeared in a very contrast area in the center of the cell. This area was not indicated on the defectogram of the original cell. A region of different contrast compared to the rest of the cell, which was situated between the laser made defects, could also be found on the LEDILIT defectogram, but it was much less distinct. Thus, it was not fully clear if this region on the FPT defectogram was in relation with the artificial defects or was caused by an inhomogeneous heating. Defects D3 and D4 were not detected by the FPT.

The laser made defects (D1-D4) damaged the emitter. These defects as well as the cracks and local shunts on the original cell influenced the photo-voltaic effect during the excitation, as it was explained in the section 3.A. Thus, all defects were very clearly indicated by the LEDILIT inspection. It was also shown that the CNR evaluation corresponded to a different intensity of the damages caused by different laser power the higher laser power caused the higher CNR of the indications of the defects. The FPT inspection did not indicate all of the artificial defects made by the laser. However, the defects made by the higher heat input (D1 and D2) as well as some of the original shunts were identified also by the FPT. It demonstrates that the FPT is not as sensitive as the LEDILIT, but it is also able to find some more significant defects.

The solar cell no. 2 was partly damaged during the manipulation before the thermographic inspection of the cell with the defects created by the laser. A breaking of a part of the cell was evident on both LEDILIT and FPT inspections results at the lower right corner of the cell. However, this damage did not have an influence of any previous results (IVC results for example) and it was not supposed that it affected the thermographic inspections.

\section{Solar cell 3}

The FPT and LEDILIT inspections results together with photos of a top and bottom side of the cell no.3 are shown in Fig. 9. The FPT defectogram was evaluated by the $1^{\text {st }}$ derivation ROOT method (AT labeling, method based on approximation and derivation of the response signal) because these results were better than results by the pulse-phase evaluation. The LEDILIT defectogram was obtained by the DPR method and phase results presentation.
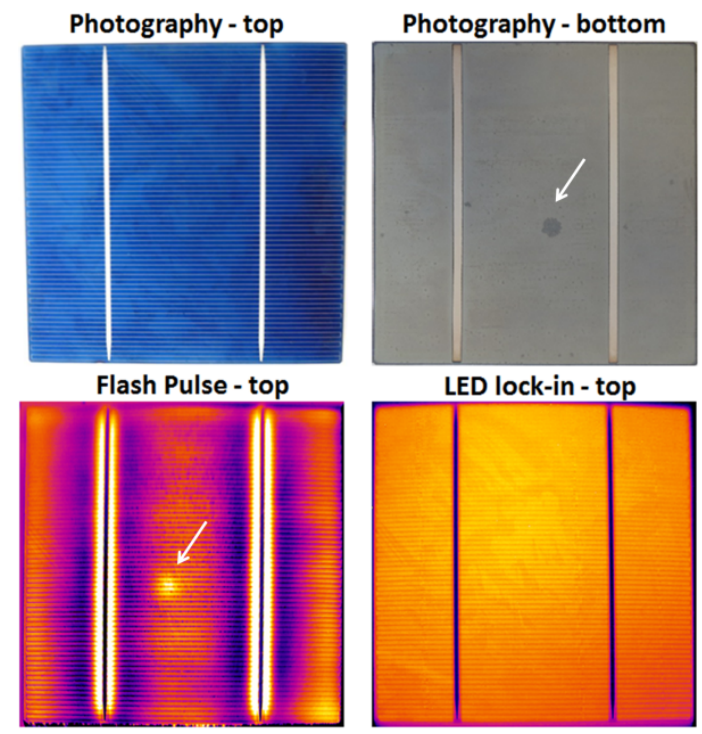

Fig. 9 The solar cell no. 3: photography of the cell from top and bottom side, FPT inspection defectogram and LEDILIT defectogram.
The thermographic inspections of the cell no. 3 brought quite different results compared to the cells no. 1 and 2. The busbars were much more distinct on the both FPT and LEDILIT defectograms, but no local shunts or cracks similar to these on the cells no. 1 and 2 were found either by the FPT or LEDILIT methods. However, an area-type indication was found out at a central part of the cell by the FPT inspection (marked by an arrow in Fig. 9, the image "Flash Pulse top"). It was discovered that it was related to an inhomogeneity at the bottom side of the cell (see Fig. 9, the image "Photography - bottom").

The inhomogeneity found by the FPT inspection on the back side of the cell was caused by some type of a liquid. The liquid was probably soaked in a bottom layer of the cell. This caused a change of thermal properties of this area, which was identified by the FPT inspection. This inhomogeneity didn't influence the photo-voltaic effect and, therefore, it was not detected by the LEDILIT inspection.

\section{Results summary}

The results of the performed experiments showed differences between the FPT and LEDILIT inspections, which were based on different properties of the excitation sources (intensity, wavelength, homogeneity) and excitation procedures (periodical vs. pulsed). Different excitation principles led to different processes during the interaction of the excitation light with the measured solar cells and thus also to different excitation mechanisms. The LEDILIT (Voc-ILIT) excitation was based on long-term periodical excitation by a relatively low-intensity source. A response of the tested cells was influenced by residual currents [8] that induced a sufficient thermal response on the defects. This effect was stronger than a direct heat excitation from the LED source. Another advantage was that it was not too complicated to make a flat light source from a number of LED diodes of different colors (i.e. different wavelengths). It followed that the source was very homogeneous and a specific combination of LED diodes could be used for a LED source wavelength range optimization.

The residual currents were induced also by the flash pulse. However, these currents were mostly not sufficient to play a significant role in a thermal response of the defects by the used experimental configuration (the used flash lamp, pulse-length etc.). The thermal response of the cells to their flash light excitation was therefore mostly a consequence of a heat transfer process caused by a direct heating by the flash lamp. It means that the response was mostly made by inhomogeneities or discontinuities, which influenced the heat transfer process. As a consequence, the small defects (local shunts) and cracks connected with the photovoltaic effect were not indicated or their indications were very weak. The more significant defects were indicated also by the FPT, however, the indications of these defects was not as distinct as at the LEDILIT inspections.

The flash-lamp was basically a more localized light source. Thus, the FPT inspections were also more sensitive to geometrical configuration imperfections. It led to excitation inhomogeneities and decreased a detectability of the small defects. On the other hand, the FPT inspection was much faster and was able to detect also an inhomogeneity on the back side of the cell. This inhomogeneity, which caused a change of thermal properties of the back side only and was not connected with a photo-voltaic effect, was not indicated by the LEDILIT inspection.

\section{CONCLUSION}

A usability of the two active thermography methods for solar cells inspection was analyzed in this work: the LED lock-in (LEDILIT) and the flash-pulse (FPT) thermography. Three solar cells were used for the experiments. The solar cells were inspected in their original state and two samples were also tested after artificial defects were made on their top surface using the laser technology. The thermographic 
measurement was accompanied by the measurement of the electrical properties (IVC) of the cells.

It was confirmed by the IVC measurement that all laser made defects had influence on the solar cells performance and were more significant than original small defects, which were indicated mostly by the LEDILIT inspections. The LEDILIT inspections detected all laser made defects and also a number of small original defects (shunts, cracks) on the cells no. 1 and 2. The more significant defects were more distinct on the defectograms. No defect was found by the LEDILIT on the solar cell no. 3, which was the same type but made by a different producer. The most significant defects only were indicated on the top side of the cells by the FPT inspections. The FPT inspection however detected an inhomogeneity on the back side of the cell no. 3, which was not connected with a photo-voltaic effect and was not indicated by the LEDILIT inspection.

It can be concluded that for defects connected with the photo-voltaic effect the LEDILIT inspection provided a better detectability than the FPT inspection. It can be therefore stated that this method is fully usable for solar cells testing. A disadvantage of this method was alonger inspection time, which was about $7 \mathrm{~min}$ for the used measurement set-up. This conclusion corresponds to other published results [3][17]. For this reason, the LEDILIT is not too suitable for a serial production testing. It was also not possible by the used LEDILIT measurement configuration to indicate defects, which were not connected with the photo voltaic effect, because the excitation source was too weak. The FPT was able to indicate more significant defects only on the top side of the cells. The photo-voltaic effect played not so significant role at the FPT inspections. That means, it could only inspect those defects, which could sufficiently affect a thermal process induced directly by a flash pulse. That is the reason why this method is not so common for solar cells testing. The advantage of the FPT was a short inspection time, which was about $10 \mathrm{~s}$ in our case. Another advantage of the FPT was that it made possible to inspect also defects at the back side of the cell, which were not connected with the photo-voltaic effect and which were not detected by the LEDILIT.

It is worth mentioning that a performance of solar cells is the most important and the well measurable property for their producers, testers or solar modules manufacturers. Inspection methods, which are able to reveal defects connected with a decay of the performance, are therefore more attractive for these users. These defects are related to the photo-voltaic effect and the LEDILIT technique is therefore more suitable for their inspections. However, some defects, which do not have a direct influence on an electric performance of solar cells, can bring problems in a future. It can, for example, lower a lifetime of the cells. It was shown in this paper that FPT methods could be more suitable for inspection of some defects, which are not connected with the photo voltaic effect.

It can be assumed that both introduced inspection methods could be optimized by an improvement of a measurement configuration (more homogeneous light source, higher power of an excitation source, more suitable wavelength of the excitation light etc.).

\section{FUNDING INFORMATION}

The work has been supported by the Ministry of Education, Youth and Sports of the Czech Republic within the OP RDI program, CENTEM project, no. CZ.1.05/2.1.00/03.0088, co-funded by the ERDF; and National Sustainability Programme I., CENTEM PLUS project, no. L01402 and also by projectSGS-2016-005.

[1] B. Burger, K. Kiefer, and C. Kost, "Photovoltaics Report," 2016

[2] J. A. Tsanakas, L. Ha, and C. Buerhop, "Faults and infrared thermographic diagnosis in operating c-Si photovoltaic modules: A review of research and future challenges," Renew. Sustain.
Energy Rev., vol. 62, no. November 2017, pp. 695-709, 2016. O. Breitenstein, "Illuminated versus dark lock-in thermography investigations of solar cells," Int. J. Nanoparticles, vol. 6, no. June 2012, pp. 81-92, 2013.

[4] M. Kontges, S. Kurtz, C. Packard, and U. Jahn, "Review of Failures of Photovoltaic Modules," 2014.

[5] J. Bauer, O. Breitenstein, J. Wagner, M. Planck, and M. Physics, "Lock-in Thermography : A Versatile Tool for Failure Analysis of Solar Cells," 40th Int. Test Conf., pp. 6-12, 2009.

[6] V. Tamrakar, S. C. Gupta, and yashwant Sawle, "Single-Diode and Two-Diode Pv Cell Modeling Using Matlab for Studying Characteristics of Solar Cell Under Varying Conditions," Electr. Comput. Eng. An Int. J., vol. 4, no. 2, pp. 67-77, 2015.

[7] O. Breitenstein, J. Bauer, K. Bothe, D. Hinken, J. Müller, W. Kwapil, M. C. Schubert, and W. Warta, "Luminescence imaging versus lock-in thermography on solar cells and wafers," Proc. 26th Eur. Photovolt. Sol. Energy Conf. Exhib., vol. 1, no. 2, pp. 1031-1038, 2011.

[8] O. Breitenstein and J. P. Rakotoniaina, "Lock In Thermography - a universal tool for local analysis of solar cells," 20th Eur. Photovolt. Sol. Energy Conf., vol. 7, no. 11, pp. 956-963, 2005.

[9] C. Meola, Ed., Infrared Thermography Recent Advances and Future Trends. BENTHAM SCIENCE PUBLISHERS, 2012.

[10] C. Ibarra-Castanedo, J. R. Tarpani, and X. P. V Maldague, "Nondestructive testing with thermography," Eur. J. Phys., vol. 34, no. 6, pp. S91-S109, 2013.

[11] M. Lizaranzu, A. Lario, A. Chiminelli, and I. Amenabar, "Nondestructive testing of composite materials by means of active thermography-based tools," Infrared Phys. Technol., vol. 71, pp. 113-120, 2015.

[12] J. Skála, M. Švantner, J. Tesař, and A. Franc, "Active thermography inspection of protective glass contamination on laser scanning heads," Appl. Opt., vol. 55, no. 34, pp. D60-D66, 2016.

[13] G. Pitarresi, "Lock-In Signal Post-Processing Techniques in InfraRed Thermography for Materials Structural Evaluation," Exp. Mech., vol. 55, no. 4, pp. 667-680, 2015.

[14] O. Breitenstein, W. Warta, and M. Langenkamp, Lock-in thermography, vol. 10. Berlin, Heidelberg, Germany: Springer Berlin Heidelberg, 2010.

[15] O. Breitenstein, J. Bauer, K. Bothe, D. Hinken, J. Muller, W. Kwapil, M. C. Schubert, and W. Warta, "Can luminescence imaging replace lock-in thermography on solar cells and wafers?," in 2011 37th IEEE Photovoltaic Specialists Conference, 2011, vol. 1, no. 2, pp. 159-167.

[16] H. Straube, M. Siegloch, A. Gerber, J. Bauer, and O. Breitenstein, "Illuminated lock-in thermography at different wavelengths for distinguishing shunts in top and bottom layers of tandem solar cells," Phys. Status Solidi Curr. Top. Solid State Phys., vol. 8, no. 4, pp. 1339-1341, 2011.

[17] S. Huth, O. Breitenstein, A. Huber, D. Dantz, U. Lambert, and F. Altmann, "Lock-In IR-Thermography - A Novel Tool for Material and Device Characterization," Solid State Phenom., vol. 82-84, pp. 741-746, Nov. 2002.

[18] V. P. Vavilov and D. D. Burleigh, "Review of pulsed thermal NDT: Physical principles, theory and data processing," NDTE Int., vol. 73, pp. 28-52, 2015.

[19] X. Maldague, F. Galmiche, and A. Ziadi, "Advances in pulsed phase thermography," Infrared Phys. Technol., vol. 43, no. 3-5, pp. $175-181,2002$.

[20] M. Švantner, Z. Veselý, and L. Muzika, "Depth limits of flash-pulse IRNDT method for low- and high-diffusivity materials," in Advanced Infrared Technology \& Applications, 2015, pp. 11-15. C. Ibarra-Castanedo, D. Gonzalez, F. Galmiche, X. P. Maldague, and $A$. Bendada, "Discrete signal transforms as a tool for processing and analyzing pulsed thermographic data," Proc. SPIE, vol. 6205, p. 12,2006

[22] D. L. Balageas, J.-M. Roche, F.-H. Leroy, W.-M. Liu, and A. M. Gorbach, "The thermographic signal reconstruction method: A 
powerful tool for the enhancement of transient thermographic images," Biocybern. Biomed. Eng., vol. 35, no. 1, pp. 1-9, 2015.

[23] M. Švantner, M. Kučera, E. Smazalová, Š. Houdková, and R.

Čerstvý, "Thermal effects of laser marking on microstructure and corrosion properties of stainless steel," Appl. Opt., vol. 55, no. 34, pp. D35-D45, Dec. 2016.

[24] X. P. V. Maldague, Theory and practice of infrared technology for nondestructive testing. Wiley, 2001.

[25] KEITHLEY, "Measuring Photovoltaic Cell I-V Characteristics with the Model 2420 SourceMeter Instrument," 2003. 